\title{
Video Article \\ Using Zebrafish Larvae to Study the Pathological Consequences of Hemorrhagic Stroke
}

\author{
Siobhan Crilly ${ }^{1}$, Alexandra Njegic ${ }^{2}$, Adrian R. Parry-Jones ${ }^{2,3}$, Stuart M. Allan ${ }^{1,3}$, Paul R. Kasher ${ }^{1,3}$ \\ ${ }^{1}$ Division of Neuroscience and Experimental Psychology, School of Biological Sciences, Manchester Academic Health Science Centre, University of Manchester

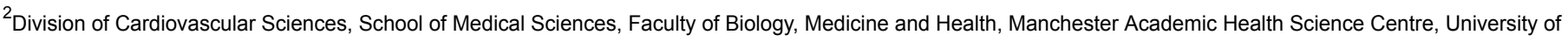 \\ Manchester \\ ${ }^{3}$ Lydia Becker Institute of Immunology and Inflammation, University of Manchester
}

Correspondence to: Paul R. Kasher at paul.kasher@manchester.ac.uk

URL: https://www.jove.com/video/59716

DOI: doi:10.3791/59716

Keywords: Neuroscience, Issue 148, Intracerebral hemorrhage, zebrafish, brain injury, stroke, neuroinflammation, animal models, pre-clinical

Date Published: 6/5/2019

Citation: Crilly, S., Njegic, A., Parry-Jones, A.R., Allan, S.M., Kasher, P.R. Using Zebrafish Larvae to Study the Pathological Consequences of Hemorrhagic Stroke. J. Vis. Exp. (148), e59716, doi:10.3791/59716 (2019).

\section{Abstract}

Despite being the most severe subtype of stroke with high global mortality, there is no specific treatment for patients with intracerebral hemorrhage $(\mathrm{ICH})$. Modelling $\mathrm{ICH}$ pre-clinically has proven difficult, and current rodent models poorly recapitulate the spontaneous nature of human $\mathrm{ICH}$. Therefore, there is an urgent requirement for alternative pre-clinical methodologies for study of disease mechanisms in ICH and for potential drug discovery.

The use of zebrafish represents an increasingly popular approach for translational research, primarily due to a number of advantages they possess over mammalian models of disease, including prolific reproduction rates and larval transparency allowing for live imaging. Other groups have established that zebrafish larvae can exhibit spontaneous $\mathrm{ICH}$ following genetic or chemical disruption of cerebrovascular development. The aim of this methodology is to utilize such models to study the pathological consequences of brain hemorrhage, in the context of preclinical $\mathrm{ICH}$ research. By using live imaging and motility assays, brain damage, neuroinflammation and locomotor function following ICH can be assessed and quantified.

This study shows that key pathological consequences of brain hemorrhage in humans are conserved in zebrafish larvae highlighting the model organism as a valuable in vivo system for pre-clinical investigation of $\mathrm{ICH}$. The aim of this methodology is to enable the pre-clinical stroke community to employ the zebrafish larval model as an alternative complementary model system to rodents.

\section{Video Link}

The video component of this article can be found at https://www.jove.com/video/59716/

\section{Introduction}

Intracerebral hemorrhage $(\mathrm{ICH})$ is the most severe sub-type of stroke associated with spontaneous cerebral vessel rupture and bleeding into the parenchyma leading to brain damage, physical disability and often death ${ }^{1}$. Despite the high mortality and morbidity rate associated with $\mathrm{ICH}^{2}$, understanding of the underpinning etiology and post-hemorrhage pathology is still lacking. As such, there are no specific treatments to prevent $\mathrm{ICH}$ or improve patient outcomes. Most of our understanding of disease biology has come from pre-clinical rodent models of $\mathrm{ICH}^{3}$, however studies to-date in these models have failed to translate any successful therapeutic to the clinic ${ }^{4,5}$. This failure may be due in part, to some limitations of these preclinical models, including the inability to easily recapitulate the spontaneous nature of human disease and the requirement for invasive surgery to generate the models in mammals 6 . Additionally, rodents pose practical problems with regards to observing the rapid onset of cellular responses to $\mathrm{ICH}$ in intact tissue. Given the lack of translation from rodent models, developing alternative models of spontaneous ICH is imperative if we are to overcome these practical problems and help identify novel drug targets.

The molecular mechanisms of vascular development are well conserved amongst vertebrates including zebrafish (Danio rerio $)^{7}$. As such, the adoption of this model organism is becoming an ever more useful mechanistic strategy for studying cerebrovascular disease ${ }^{8}$. A number of zebrafish models have been generated which recapitulate phenotypes associated with stroke-related conditions ${ }^{9,10,11,12}$. The use of zebrafish larvae to investigate disease pathogenesis offers both practical and scientific advantages over mammalian models ${ }^{8}$. This includes high reproduction rates, rapid development and larval transparency that allows for intravital imaging without the invasive constraints associated with rodents. Coupling these advantages with the wide range of transgenic reporter lines available within the zebrafish research community amounts to a powerful in vivo approach for studying disease biology, not yet utilized for studying the pathological consequences of ICH.

The injury response to blood in the brain is biphasic ${ }^{13}$; the primary insult causes neuronal death and cell necrosis, which then initiates a secondary wave of damage that is induced by innate immune activation. The second phase of brain injury, in particular the neuroinflammatory component, is considered a realistic target for future drug treatment ${ }^{13}$. Spontaneous and cerebral-specific hemorrhages have been described in 
zebrafish larvae previously ${ }^{14,15,16,17,18,19}$. Two such models are the use of atorvastatin (ATV) at 24 h post-fertilization (hpf) to inhibit the HMGCR pathway and cholesterol biosynthesis ${ }^{14}$, and a bubblehead (bbh) mutant which express a hypomorphic mutation in the arhgef7 gene, $\beta$ pix, and subsequently inhibits actin remodeling for tight endovascular junctions ${ }^{18}$. These models exhibit spontaneous cerebral-specific blood vessel rupture at the onset of circulation ( $\sim 33 \mathrm{hpf})$. Recently, we have characterized these models further to reveal that key aspects of the brain injury response is conserved between humans and zebrafish larvae ${ }^{20}$. This study demonstrates the methodology required to obtain and visualize spontaneous brain hemorrhages in zebrafish larvae and how to quantify brain injury, and locomotor and neuroinflammatory phenotypes that relate to the human condition. These data and techniques support the use of this model species as a valuable complementary system for preclinical $\mathrm{ICH}$ research.

\section{Protocol}

Zebrafish were raised and maintained at The University of Manchester Biological Services Unit under standard conditions as previously described $^{21}$. Adult zebrafish husbandry was approved by the University of Manchester Animal Welfare and Ethical Review Board. All experiments were performed in accordance with U.K. Home Office regulations (PPL:P132EB6D7).

NOTE: Transgenic lines used in this study include macrophage-specific lineage mpeg1:mCherry (constructed in-house as previously described 22 ), neutrophil-specific mpo:GFP ${ }^{23}$,erythroid-specific gata1:dsRed ${ }^{24}$ and ubiq:secAnnexinV-mVenus, a reporter for cell death (re-derived in house $\left.{ }^{25}\right)$ on wild-type, nacre $\left(\right.$ mitfa $\left.^{\text {w2/w2 }}\right)$ and mutant $\left(b b h^{\mathrm{m} 292}\right)$ backgrounds. Figure 1 shows the experimental timeline.

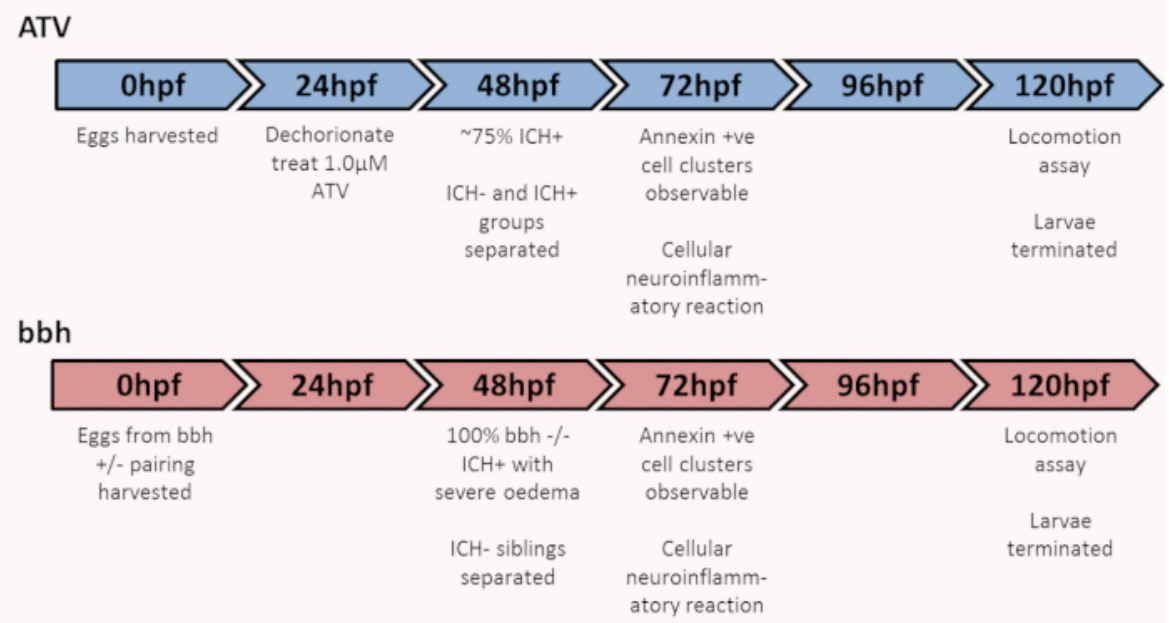

Figure 1: Graphic of experimental timeline to characterize brain injury, locomotor and neuroinflammatory outcomes. ICH, intracerebral hemorrhage; bbh, bubblehead. Figure has been reproduced from Crilly et al. ${ }^{20}$ with permission under a Creative Commons license. Please click here to view a larger version of this figure.

\section{Day 0: Egg production and collection}

1. Collect fertilized embryos from natural spawning in breeding boxes produced from 1 male and 1-2 female adult zebrafish.

NOTE: For atorvastatin protocol any wildtype/transgenic animals can be used however hemorrhage rates differ slightly between strains.

2. Incubate 100 embryos at $28^{\circ} \mathrm{C}$ in standard E3 embryo medium per Petri dish and stage according to standard guidelines ${ }^{26}$.

3. At $\sim 6$ hours post fertilization (hpf) remove dead and unfertilized embryos from the dish using a Pasteur pipette.

\section{Day 1: Atorvastatin treatment at $24 \mathrm{hpf}$}

1. Dechorionate embryos for atorvastatin treatment using sharp ultra thin dissection forceps ${ }^{27}$. Numbers required for experimentation can be adjusted accordingly.

2. Add $30 \mathrm{~mL}$ of E3 embryo medium to two clean Petri dishes. Use one dish for 100 embryos.

NOTE: If plates are designed for cell culture, dechorionated zebrafish at this early stage often stick to the bottom. To avoid this, rinse the plates thoroughly in clean water before use.

3. Remove $60 \mu \mathrm{L}$ of embryo water from the treatment plate and add $60 \mu \mathrm{L}$ of $0.5 \mathrm{mM}$ atorvastatin (ATV). At a $0.5 \mathrm{mM}$ stock concentration, the above dilution will result in a final concentration of $1 \mu \mathrm{M}$ which will result in $\sim 20 \%$ of larvae non-hemorrhaged (ICH-) and $\sim 80 \%$ of larvae hemorrhaged $(\mathrm{ICH}+)$. Use the other plate for untreated controls

NOTE: Atorvastatin is solubilized in distilled water $(3 \mathrm{mg}$ into $10 \mathrm{~mL})$ to make a $0.5 \mathrm{mM}$ stock solution. Incubate overnight at room temperature in the dark with agitation as solubilization takes some time. Complete solubilization can take up to 1 week. Do not use DMSO. Solution is aliquoted and stored at $-20^{\circ} \mathrm{C}$. Do not freeze thaw.

4. Using a Pasteur pipette, transfer 100 embryos in as little water as possible to the treatment plates.

5. Incubate the plates at $28^{\circ} \mathrm{C}$.

NOTE: ICH will occur between 33 and $48 \mathrm{hpf}$. Atorvastatin does not need to be removed as incubation longer than $24 \mathrm{~h}$ does not cause any further developmental issues. 


\section{Day 2: Separating $\mathrm{ICH}-$ and $\mathrm{ICH}+$ populations at $50 \mathrm{hpf}$}

1. Separate $\mathrm{ICH}+$ fish from $\mathrm{ICH}$ - populations and transfer to new dishes for ease.

1. If using the ATV model at a concentration of $1 \mu \mathrm{M}, 75-100 \%$ of larvae will exhibit hemorrhage $(\mathrm{ICH}+)$ at this time point. NOTE: The response of the larvae differs between strains, if larvae are not hemorrhaging at the desired frequencies, use a fresh batch of atorvastatin or a higher concentration. If larvae have not exhibited hemorrhage by $48 \mathrm{hpf}$ then consider them ICH-.

2. If using the bbh model, all homozygous mutants will exhibit hemorrhage by $48 \mathrm{hpf}$. If using a heterozygous incross, the ICHheterozygous and wildtype siblings can be used as control animals for experiments.

2. If necessary, anaesthetize the larvae by adding $0.02 \% \mathrm{MS} 222$ to the E3 media. Using a Pasteur pipette, sort the larvae for presence of blood in the head into fresh E3 media. See Figure 2.

NOTE: Blood in the head may appear in the fore, mid or hindbrain or in combination, and bleed volume can vary between animals. In the bbh mutants, $\mathrm{ICH}$ is often associated with severe edema recognizable by larger heads, wider space between the eyes and a more diffuse bleed. However not all ICH+ bbh larvae exhibit edema.

\section{$\mathrm{ICH}-$}
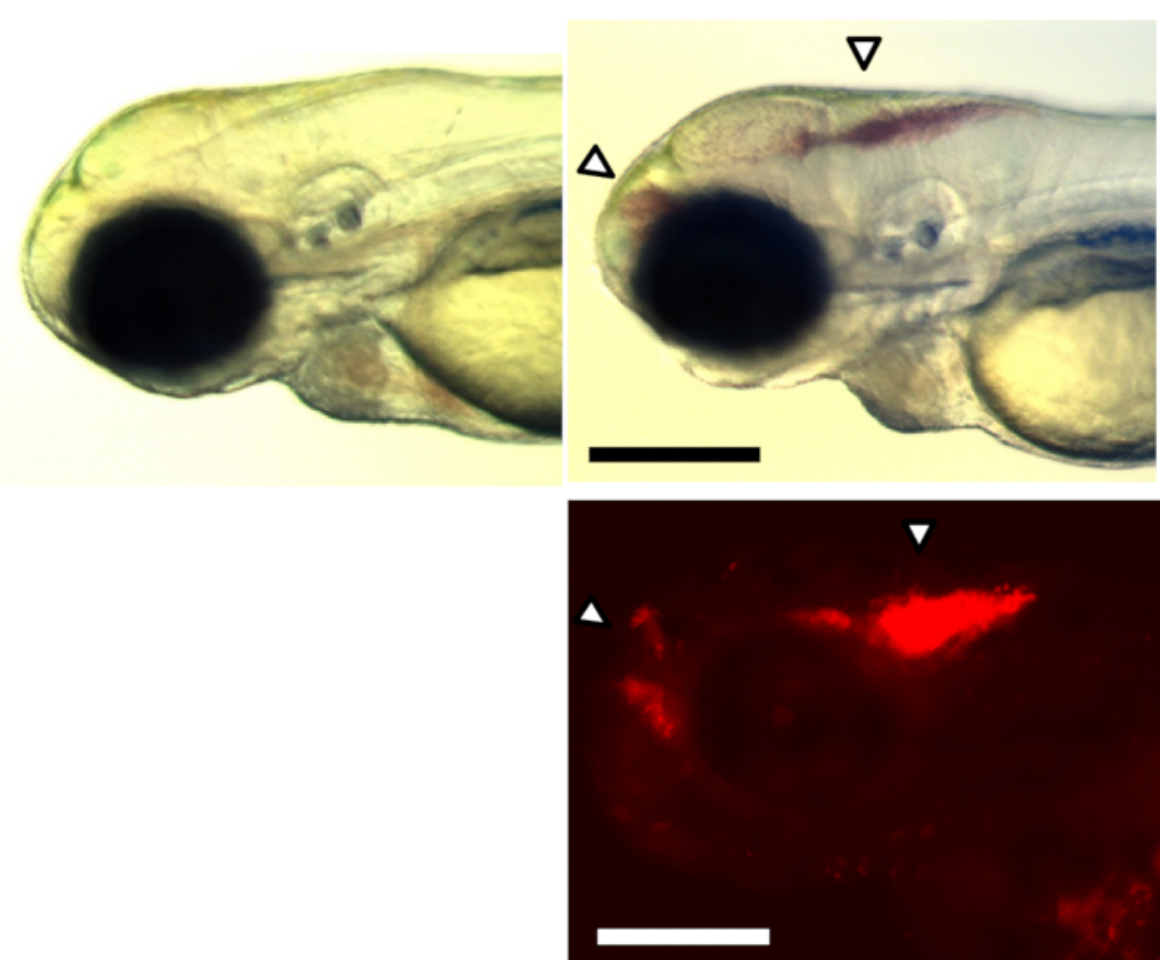

Figure 2: $\mathrm{ICH}+$ brain hemorrhage phenotypes. Examples of larval ICH phenotypes maintained on a transgenic gata1:DsRed reporter nacre background observed with a brightfield stereomicroscope (top panels) and fluorescence (bottom panel) at $\sim 48 \mathrm{~h}$ post-fertilization. No hemorrhages were observed in ICH-larvae (left panels). A distinct accumulation of red blood cells in the forebrain and hindbrain (arrows) were observed in $\mathrm{ICH}+$ larvae (right panels). Scale bars represent $250 \mu \mathrm{m}$. Figure has been reproduced from Crilly et al. ${ }^{20}$ with permission under a Creative Commons license. Please click here to view a larger version of this figure.

\section{Day 3: Cell death and leukocyte analysis at $72 \mathrm{hpf}$}

1. Screen the larvae using a fluorescence microscope to ensure the expression of fluorescent protein.

NOTE: In this study, transgenic ubiq:secAnnexinV-mVenus larvae were used to report brain cell death and double transgenic mpo:GFP;mpeg1:mCherry or ubiq:secAnnexinV-mVenus;mpeg1:mCherry used for leukocyte analysis.

2. Fill the lightsheet mounting chamber with E3 media containing $0.02 \% \mathrm{MS} 222$.

3. Anaesthetize the larvae using $0.02 \%$ MS222. Transfer larvae for mounting $(n=1-6)$ to a dry Petri dish surface in a single droplet. Remove as much liquid as possible.

NOTE: $1.5 \%$ low melt agarose is prepared using $0.15 \mathrm{~g}$ of low melt agarose dissolved in $10 \mathrm{~mL}$ of E3 medium without methylene blue in a microwave and kept at $45^{\circ} \mathrm{C}$ until use.

4. Add a drop of $1.5 \%$ low-melt agarose (maintained as liquid in a $45^{\circ} \mathrm{C}$ heat block) to the larvae and using a $800 \mu \mathrm{m}$ mounting capillary, and draw the larvae up head first. If positioning is not accurate the larvae can be expelled from the agarose and mounted again. Leave the capillary to cool before inserting into the lightsheet chamber.

NOTE: Alternatively, a confocal microscope could be used for this procedure. For this, larvae should be mounted laterally in agarose on a glass bottom dish.

5. Acquire $z$-stack images of the head between the eye lenses $(\sim 300 \mu \mathrm{m})$ and process to maximum intensity projection image. 
1. Analyze brain region from images collected for total number of fluorescent cells and total intensity fluorescence ${ }^{20}$.

6. Create a time lapse video of multiple projection composites over 18-24 h to track leukocyte mobility and interaction with dying cells. NOTE: If long term live imaging is performed, only one larva is mounted in the capillary.

7. When imaging is completed expel the larvae from the mounting capillary into a lethal overdose of $4 \%$ MS 222 to euthanize.

\section{Day 3: Selecting larvae for motility assay at $72 \mathrm{hpf}$}

1. Anaesthetize larvae in the petri dishes with $0.02 \% \mathrm{MS} 222$.

2. Randomly select $n=24$ larvae for motility assay and transfer into fresh E3 media and allow animals to recover from anesthetic. NOTE: Anesthetic at this point removes selection bias for slow swimmers that are easy to catch.

\section{Day 3-5: Assaying locomotion at 72,96 and $120 \mathrm{hpf}$}

1. Transfer larvae selected at $72 \mathrm{hpf}$ into E3 medium without methylene blue. NOTE: For assaying at $3 \mathrm{dpf}$ allow the larvae ample time to recover from anesthetic $(>1 \mathrm{~h})$.

2. Plate one larva in $1 \mathrm{~mL}$ per well of a 24 -well plate using a pipette. NOTE: Cut the end of the pipette tip to avoid damaging the larvae. Plate size can be changed according to experimental design.

3. Load plates into the camera chamber and assay motion for $10 \mathrm{~min}$ using a white light startle routine to increase spontaneous swimming. NOTE: Swimming behavior was tracked using a camera chamber and tracking software (see the Table of Materials).

4. Repeat experiment with the same larvae at 96 and $120 \mathrm{hpf}$. Move larvae from individual housing in assay plate to a Petri dish and incubate at $28^{\circ} \mathrm{C}$ in between assays.

5. At assay completion, euthanize larvae in a lethal overdose of $4 \% \mathrm{MS} 222$.

\section{Representative Results}

Assessment of brain cell death using transgenic ubiq:secAnnexinV-mVenus results in clear definitive clusters of dying cells in ICH+ larvae in both ATV and bbh models that are absent in all ICH- larvae (Figure 3). Clusters recede before $96 \mathrm{hpf}$. Through image analysis, bleeding is associated with a significant two-fold increase in total intensity of fluorescence signal in the brain, indicating marked cell death.

A neuroinflammatory response is identified in $\mathrm{ICH}+$ larvae by significantly increased numbers of $m p e g 1$ positive macrophage cells in the brain. The number of total mpo positive neutrophil cells also increased however this did not reach statistical significance (Figure 4). The morphology of the mpeg1 positive macrophages can also be seen to change in $\mathrm{ICH}+$ larvae as the cells adopt an active, rounded, amoeboid shape. These activated rounded cells can also be monitored over time to show an increased phagocytic response of the ubiq:secAnnexinV-mVenus expressing dying cells in $\mathrm{ICH}+$ larvae (Figure 5). mpeg1 positive macrophages exhibiting ramified processes were categorized as inactive.

Brain hemorrhage is associated with a significant decrease in motility at 72 and $96 \mathrm{hpf}$ in comparison to ICH-sibling controls in both bbh and ATV models (Figure 6). Motility at $120 \mathrm{hpf}$ recovers to near baseline levels. There are often differences in baseline motility between egg clutches and strains and so comparison should be made to $\mathrm{ICH}$ - controls every time. 
A
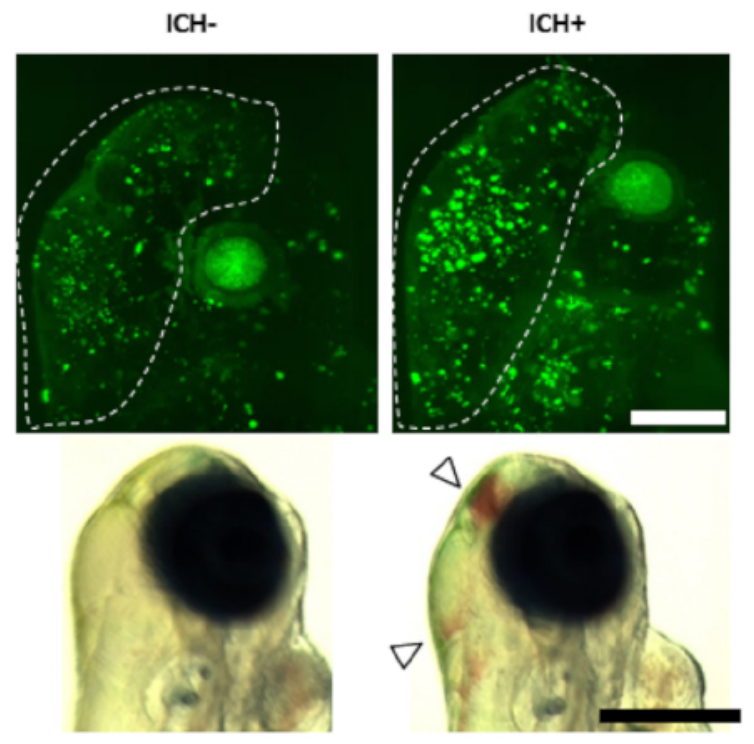

B

ATV

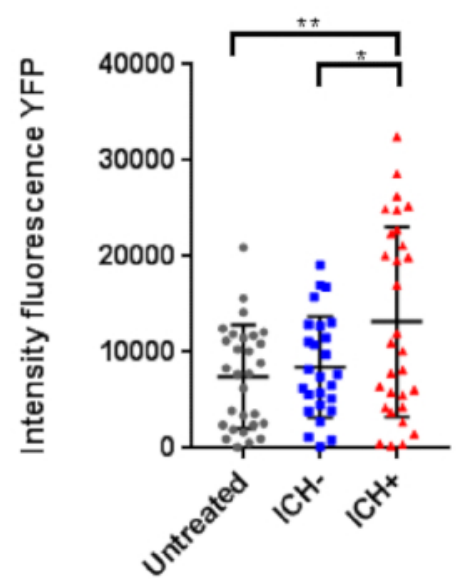

C

\section{bbh}

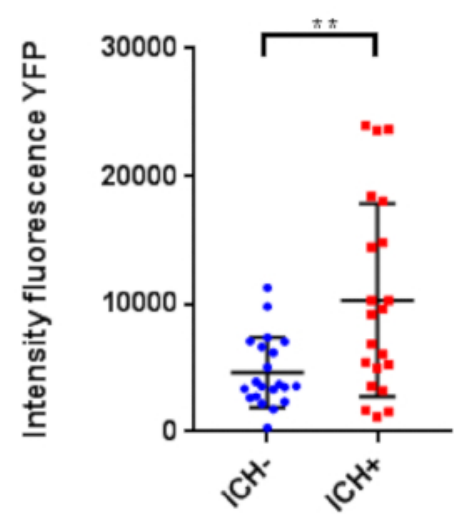

Figure 3: Intracerebral hemorrhage (ICH) in zebrafish larvae results in a quantifiable brain injury. (A) Representative images of the brain injury phenotype in $\mathrm{ICH}+$ larvae (right panels), in comparison to $\mathrm{ICH}$ - siblings (left panels), at $72 \mathrm{hpf}$. Brightfield images (bottom panels, scale bar $=250 \mu \mathrm{m}$ ) demonstrate the presence of brain bleeds (arrows) in $\mathrm{ICH}+$ larvae. Fluorescent microscopy was performed to visualize cell death in the ubiq:secAnnexinV-mVenus reporter line (top panels, scale bar $=100 \mu \mathrm{m}$ ). Clusters of dying cells were observed in peri-hematomal regions. Images were cropped to brain-only regions and analyzed for total green fluorescence intensity in round particles larger than 30 pixels in diameter (white line) using the macro in Supplementary File 1. (B) Quantification of fluorescence signal in the brains of untreated, ICH- and ICH+ larvae obtained through the ATV model ( $\mathrm{n}=12$ per group; 3 independent replicates) at $72 \mathrm{hpf}$. Significant differences were observed when comparing $\mathrm{ICH}+$ with untreated $\left({ }^{* *} \mathrm{p}=0.004\right)$ and with $\mathrm{ICH}-\left({ }^{*} p=0.03\right)$ siblings. (C) Quantification of fluorescence signal as a read out for annexinV binding in the brains of $\mathrm{ICH}$ - and $\mathrm{ICH}+$ larvae obtained through the bubblehead (bbh) model ( $\mathrm{n}=12$ per group; 2 independent replicates) at $72 \mathrm{hpf}$. Graphs show SD from the mean. A significant difference in mVenus fluorescence was observed between $\mathrm{ICH}+$ and $\mathrm{ICH}$ - age-matched siblings $\left({ }^{* *} p=0.002\right)$. Figure has been reproduced from Crilly et al ${ }^{20}$ with permission under a Creative Commons license. Please click here to view a larger version of this figure. 
A

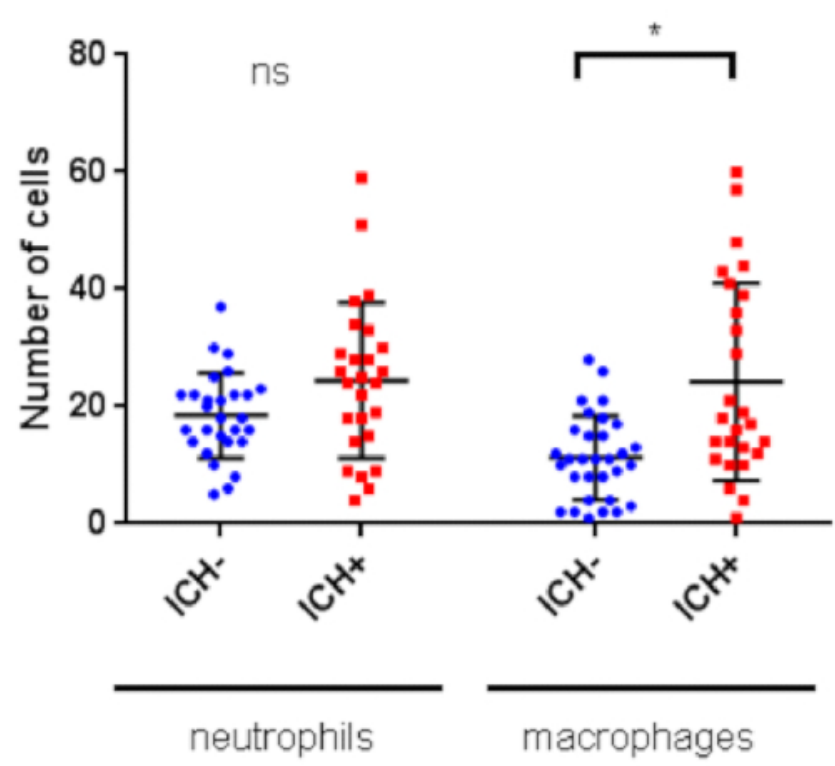

Figure 4: Intracerebral hemorrhage (ICH) initiates an innate cellular immune response in the zebrafish larval brain. Numbers of leukocytes quantified within the brain regions previously described for mpo:GFP;mpeg1:dsRed double transgenic larvae ( $n=8$ per group; 2 independent replicates) at $72 \mathrm{hpf}$ reveals a significant increase in macrophages ( $\left.{ }^{*} p=0.01\right)$, but not neutrophils $(p=0.5)$, in response to ICH. Figure has been reproduced from Crilly et al. ${ }^{20}$ with permission under a Creative Commons license. Please click here to view a larger version of this figure. 

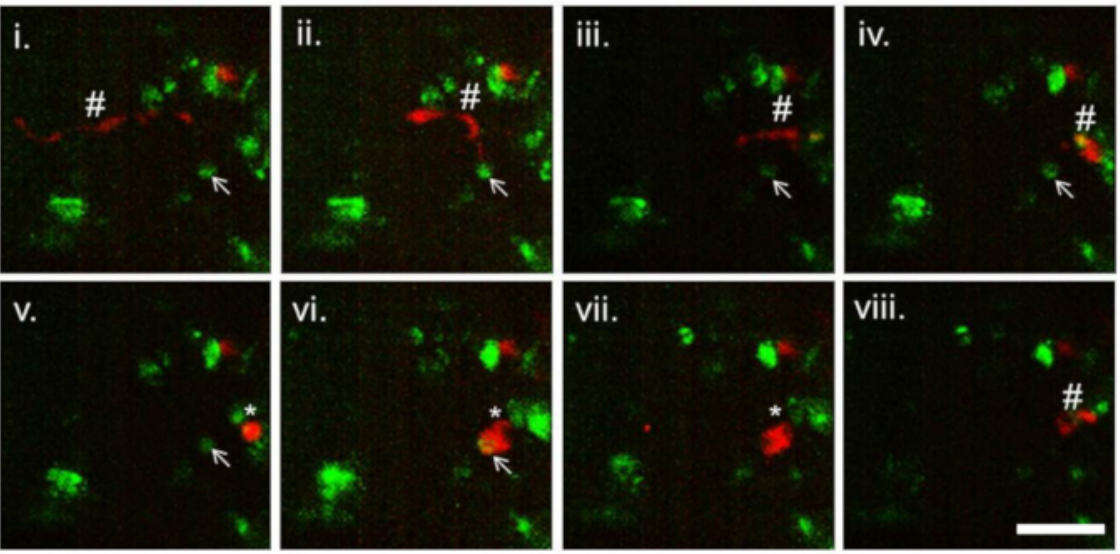

$\mathrm{ICH}-$

$\mathrm{ICH}+$
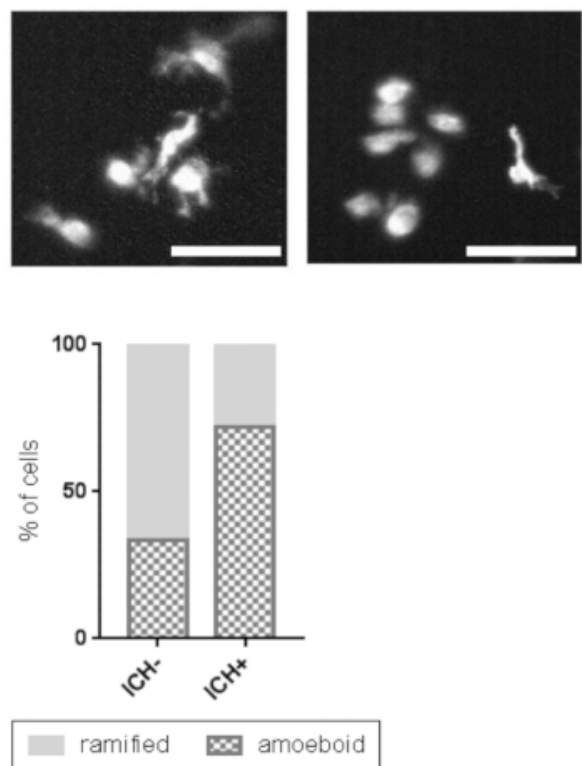

Figure 5: Activated macrophage cells show a phagocytic response to the brain lesion. (A) Representative time-lapse stills ${ }^{20}$ showing a ramified patrolling macrophage migrating towards an annexin $V$ positive cell ( $\mathrm{i}-\mathrm{vi}$ ). Stills are obtained from a series of images taken of the whole brain using a 20x objective. Scale bar represents $50 \mu \mathrm{m}$. The macrophage acquired an amoeboid morphology ( $\mathrm{v}$ ) before phagocytosing the annexinV-positive cell (vi, vii). After phagocytosis the macrophage resumes a ramified morphology and migrates away and the annexinVpositive cell can no longer be seen (viii). Ramified macrophage (\#), annexinV positive cell (arrow), amoeboid macrophage $\left({ }^{*}\right)$ are indicated. (B) Representative images of mpeg1-positive cells in the $\mathrm{ICH}$ - and $\mathrm{ICH}+$ larval brain exhibiting amoeboid and ramified morphologies. Scale bars represent $50 \mu \mathrm{m}$. (C) An increased proportion of amoeboid (phagocytic) and decreased proportion of ramified (inactive) macrophages was observed in $\mathrm{ICH}$ - brains in comparison to $\mathrm{ICH}$ - siblings. Figure has been reproduced from Crilly et al. ${ }^{20}$ with permission under a Creative Commons license. Please click here to view a larger version of this figure. 


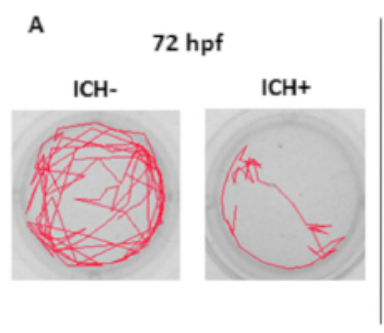

B

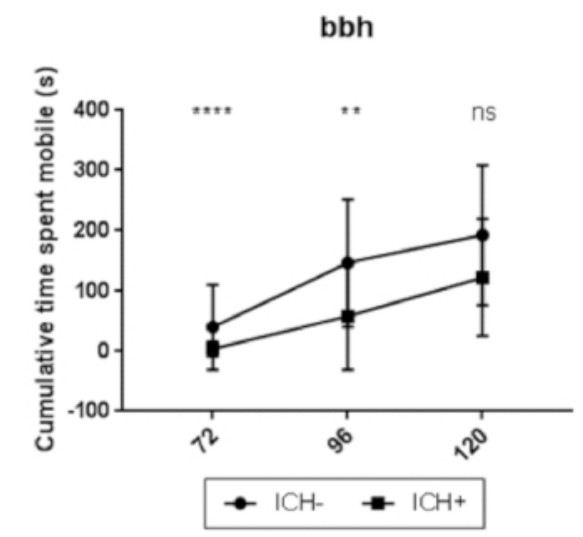

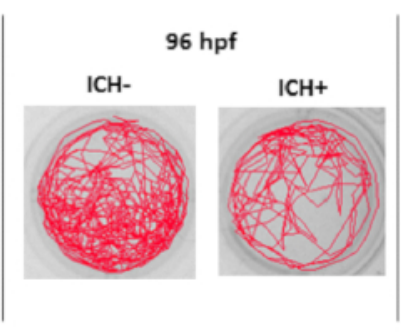

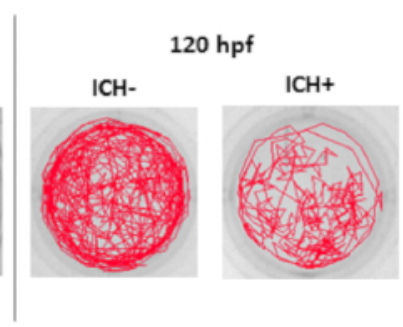

C

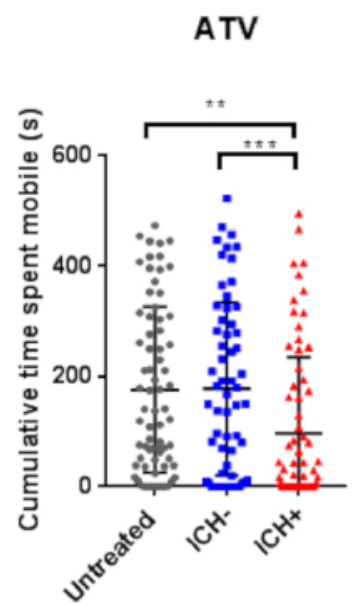

Figure 6: ICH-induced brain injury results in a quantifiable locomotor deficit in zebrafish larvae. (A) Representative examples of the swimming tracks in $\mathrm{ICH}$ - and $\mathrm{ICH}+$ bbh larvae at 72,96 and $120 \mathrm{hpf}$. (B) $\mathrm{ICH}+$ larvae exhibited a significant decrease in the cumulative time spent mobile during the $10 \mathrm{~min}$ recording period at both 72 and $96 \mathrm{hpf}$. Significance was lost at the 120 hpf time point potentially alluding to recovery from brain injury $\left(n=24\right.$ larvae per group; 3 independent replicates; ${ }^{\star * \star *} p=0.00006 ;{ }^{* \star} p=0.003 ; n s$ : $\left.p=0.08\right)$. (C) Quantification of cumulative time spent moving in untreated and ATV-treated ICH- and ICH+ larvae at $120 \mathrm{hpf}$. ICH+ larvae exhibited a significant decrease in the cumulative time spent mobile during the $10 \mathrm{~min}$ recording period. Three technical replicates $(\mathrm{n}=24$ larvae per group) were used to calculate $\mathrm{SD}$ from the mean $\left({ }^{* * *} p=0.00004,{ }^{* *} p=0.0003\right)$. Figure has been reproduced from Crilly et al. ${ }^{20}$ with permission under a Creative Commons license. Please click here to view a larger version of this figure.

Supplementary File 1. Please click here to download this file.

\section{Discussion}

This study shows that ICH in zebrafish larvae induces a brain injury response that recapitulates key aspects of the human condition that can be systematically assayed and quantified. Zebrafish offer a consistent and reproducible model of spontaneous ICH which will assist with future drug intervention studies focused on targeting blood-induced brain injury, rather than preventing vessel rupture ${ }^{17,28}$. Indeed, given the rapid nature of disease onset akin to the clinical situation, such an approach offers exciting prospects for successful translation in the future.

Some limitations are associated with the use of zebrafish larvae, such as the use of a developing system and taxonomic rank, however the practical and scientific advantages of this model must be considered to offer new insights into ICH. No surgery is required to initiate a haemorrhage or to monitor cellular processes over extended periods of time after injury. High fecundity of zebrafish pairings generate easily accessible and large sample sizes, and due to the fast development of the larvae the experimental timeline is significantly reduced compared to rodent studies 29,30 .

Currently these models are fit to use for elucidating the immediate pathological and immunological response to spontaneous ICH in the brain of live intact animals. Potentially, this model can be adapted for medium-high throughput drug screens for ICH therapies, whether preventative or recovery promoting. As such, the post-ICH pathologies presented in this study represent an alternative, complementary platform for pre-clinical $\mathrm{ICH}$ research.

\section{Disclosures}

The authors have nothing to disclose.

\section{Acknowledgments}

We would like to thank Dr David Spiller and the University of Manchester Systems Microscopy Core Facility for use of the equipment, Prof.

Richard Baines for the use of DanioVision and Dr. Jack Rivers-Auty for statistical consultation. The bbh line was kindly shared by Nicole Munsie 
from Dr. Sarah Child's lab at the University of Calgary. We also thank Prof. Stephen Renshaw, Dr. Adam Hurlstone, Dr. Andrew Badrock and Dr. Helen Young for fish lines and equipment.

This study was supported by the NC3Rs (NC/N002598/1), Stroke Association (TSA LECT 2017/02), ERA-NET NEURON (MR/M501803/1) and The British Heart Foundation (FS/15/67/32038). We are also particularly thankful to The Natalie Kate Moss Trust and the University of Manchester Faculty of Biology, Medicine and Health for their continued financial support.

\section{References}

1. An, S. J., Kim, T. J., Yoon, B.-W. Epidemiology, risk factors, and clinical features of intracerebral hemorrhage: an update. Journal of stroke. 19 (1), 3 (2017)

2. WHO. The top 10 causes of death. <http://www.who.int/mediacentre/factsheets/fs310/en/> (2017).

3. Casals, J. B.et al. The use of animal models for stroke research: a review. Comparative medicine. 61 (4), 305-313 (2011).

4. Kellner, C. P., Connolly, E. S. Neuroprotective Strategies for Intracerebral Hemorrhage Trials and Translation. Stroke. 41 (10 suppl 1), S99S102 (2010)

5. Kirkman, M. A., Allan, S. M., Parry-Jones, A. R. Experimental intracerebral hemorrhage: avoiding pitfalls in translational research. Journal of Cerebral Blood Flow and Metabolism. 31 (11), 2135-2151 (2011).

6. ASPA. 1986 amendments. (2012).

7. Butler, M. G., Gore, A. V., Weinstein, B. M. in Methods in cell biology. Vol. 105 137-161 Elsevier, (2011).

8. Walcott, B. P., Peterson, R. T. Zebrafish models of cerebrovascular disease. Journal of Cerebral Blood Flow \& Metabolism. 34 (4), $571-577$ (2014).

9. Liu, C.et al. Macrophages mediate the repair of brain vascular rupture through direct physical adhesion and mechanical traction. Immunity. $\mathbf{4 4}$ (5), 1162-1176 (2016).

10. Kasher, P. R.et al. Characterization of samhd1 morphant zebrafish recapitulates features of the human type I interferonopathy AicardiGoutieres syndrome. The Journal of Immunology. 194 (6), 2819-2825 (2015).

11. Wen, J.et al. Mutation of rnf213a by TALEN causes abnormal angiogenesis and circulation defects in zebrafish. Brain research. 1644 70-78 (2016).

12. Eisa-Beygi, S., Rezaei, M. Etiology of intracerebral hemorrhage (ICH): novel insights from Zebrafish embryos. International Journal of Developmental Biology. 60 (4-5-6), 119-126 (2016).

13. Mracsko, E., Veltkamp, R. Neuroinflammation after intracerebral hemorrhage. Frontiers in cellular neuroscience. 8388 (2014).

14. Eisa-Beygi, S., Hatch, G., Noble, S., Ekker, M., Moon, T. W. The 3-hydroxy-3-methylglutaryl-CoA reductase (HMGCR) pathway regulates developmental cerebral-vascular stability via prenylation-dependent signalling pathway. Developmental biology. 373 (2), $258-266$ (2013).

15. Shen, M.et al. Discovery of Rho-kinase inhibitors by docking-based virtual screening. Molecular BioSystems. 9 (6), $1511-1521$ (2013).

16. Huang, B.et al. Tanshinone I prevents atorvastatin-induced cerebral hemorrhage in zebrafish and stabilizes endothelial cell-cell adhesion by inhibiting VE-cadherin internalization and actin-myosin contractility. Pharmacological research. (2017).

17. Li, S.et al. Discovery of a ROCK inhibitor, FPND, which prevents cerebral hemorrhage through maintaining vascular integrity by interference with VE-cadherin. Cell death discovery. 317051 (2017).

18. Liu, J.et al. A $\beta$ Pix-Pak2a signaling pathway regulates cerebral vascular stability in zebrafish. Proceedings of the National Academy of Sciences. 104 (35), 13990-13995 (2007).

19. ten Klooster, J. P., Jaffer, Z. M., Chernoff, J., Hordijk, P. L. Targeting and activation of Rac1 are mediated by the exchange factor $\beta$-Pix. The Journal of cell biology. 172 (5), 759-769 (2006).

20. Crilly, S.et al. Using zebrafish larval models to study brain injury, locomotor and neuroinflammatory outcomes following intracerebral haemorrhage. F1000Research. 7 (2018).

21. Westerfield, M. The zebrafish book: a guide for the laboratory use of zebrafish. (2000).

22. Ellett, F., Pase, L., Hayman, J. W., Andrianopoulos, A., Lieschke, G. J. mpeg1 promoter transgenes direct macrophage-lineage expression in zebrafish. Blood. 117 (4), e49-e56 (2011).

23. Renshaw, S. A.et al. A transgenic zebrafish model of neutrophilic inflammation. Blood. 108 (13), $3976-3978$ (2006).

24. Traver, D.et al. Transplantation and in vivo imaging of multilineage engraftment in zebrafish bloodless mutants. Nature immunology. 4 (12), 1238 (2003).

25. Morsch, M.et al. In vivo characterization of microglial engulfment of dying neurons in the zebrafish spinal cord. Frontiers in cellular neuroscience. 9 (2015).

26. Kimmel, C. B., Ballard, W. W., Kimmel, S. R., Ullmann, B., Schilling, T. F. Stages of embryonic development of the zebrafish. Developmental dynamics. 203 (3), 253-310 (1995).

27. Liang, J. O. Zebrafish in the Classroom. <http://www.zfic.org> (2011).

28. Yang, R.et al. Miconazole protects blood vessels from MMP9-dependent rupture and hemorrhage. Disease Models \& Mechanisms. 10 (3), 337-348 (2017).

29. Andaluz, N., Zuccarello, M., Wagner, K. R. Experimental animal models of intracerebral hemorrhage. Neurosurgery Clinics of North America. 13 (3), 385-393 (2002).

30. Rosenberg, G. A., Mun-Bryce, S., Wesley, M., Kornfeld, M. Collagenase-induced intracerebral hemorrhage in rats. Stroke. 21 (5), $801-807$ (1990). 